\title{
Heads Up! A Novel Provocative Maneuver to Guide Acute Ischemic Stroke Management
}

\author{
Latisha K. Ali $^{a}$ Julius K. Weng ${ }^{a, b}$ Sidney Starkman ${ }^{a, b}$ Jeffrey L. Saver ${ }^{a}$ \\ Doojin Kim $^{a}$ Bruce Ovbiagele ${ }^{f}$ Brian H. Buck ${ }^{g}$ Nerses Sanossian ${ }^{\text {e }}$ \\ Paul Vespa ${ }^{a, c}$ Oh Young Bang ${ }^{\text {h }}$ Reza Jahan ${ }^{a, d}$ Gary R. Duckwiler ${ }^{a, d}$ \\ Fernando Viñuela ${ }^{i}$ David S. Liebeskind ${ }^{a}$

\begin{abstract}
Departments of a Neurology, ${ }^{b}$ Emergency Medicine, ${ }^{c}$ Neurosurgery and ${ }^{d}$ Interventional Neuroradiology, UCLA Stroke Center, David Geffen School of Medicine at UCLA, and e Department of Neurology, University of Southern California, Keck School of Medicine, Los Angeles, Calif., and ${ }^{\mathrm{f}}$ Department of Neurology, Medical University of South Carolina, Charleston, S.C., USA; ${ }^{9}$ Department of Neurology, University of Alberta, Edmonton,

Alta., Canada; ' Samsung Medical Center, Sungkyunkwan University, Seoul, South Korea;
\end{abstract} \\ iAméricas Medical City, Rio de Janeiro, Brazil
}

\section{Key Words}

Cerebral blood flow · Collaterals · Endovascular stroke therapy · Ischemic stroke

\begin{abstract}
Background: A common dilemma in acute ischemic stroke management is whether to pursue recanalization therapy in patients with large vessel occlusions but minimal neurologic deficits. We describe and report preliminary experience with a provocative maneuver, i.e. 90-degree elevation of the head of bed for $30 \mathrm{~min}$, which stresses collaterals and facilitates decisionmaking. Methods: A prospective cohort study of $<7.5 \mathrm{~h}$ of acute anterior circulation territory ischemia patients with minimal deficits despite middle cerebral artery (MCA) or internal carotid artery (ICA) occlusive disease. Results: Five patients met the study entry criteria. Their mean age was 78.4 years (range 65-93). All presented with substantial deficits (median NIHSS score 11, range 5-22), but improved while in supine position during initial imaging to normal or near-normal (NIHSS score 0-2). MRA showed persistent M1 MCA occlusions in 4, critical ICA stenosis or occlusion in 1 , and substantial perfusion-diffusion mismatch in all. To evaluate the potential for eventual collateral failure, patients were placed in a head of bed upright posture. Mean arterial pressure and heart rate were unchanged. Two showed no neurologic worsening and were treated with supportive care with excellent final outcome. Three showed worsening, including recurrent hemiparesis and aphasia at the 6th, recurrent aphasia at the $23 \mathrm{rd}$, and recurrent hemineglect at the 15th upright minute. These 3 underwent endovascular
\end{abstract}


recanalization therapies with successful reperfusion and excellent final outcome. Conclusion: The 'Heads Up' test may be a useful, simple maneuver to assess the risk of collateral failure and guide the decision to pursue recanalization therapy in acute cerebral ischemia patients with minimal deficits despite persisting large cerebral artery occlusion.

(C) 2016 S. Karger AG, Basel

\section{Introduction}

Spontaneous improvements or deterioration related to dynamic changes in cerebral perfusion can occur as part of the natural history of acute ischemic stroke. These may be associated with spontaneous thrombolysis, microembolism, reocclusion, thrombus propagation and fluctuations in the degree of collateral flow [1-4]. A common dilemma in acute ischemic stroke management is whether to pursue recanalization therapy in patients with large vessel occlusions but rapidly improving and/or mild neurologic symptoms (RIMS) [5]. The current guidelines for administering intravenous tissue plasminogen activator distinguish RIMS as a relative exclusion criterion to treatment [6]. However, data suggest that $25-29 \%$ of patients eligible for acute recanalization therapy who are excluded as a result of RIMS may be at risk for clinical deterioration with poor neurologic and functional outcomes [7-9].

Persisting large vessel occlusion identifies a subset of RIMS patients who are at high risk for subsequent neurologic deterioration $[5,10]$. These patients are exquisitely dependent on adequate collateral flow and hemodynamic stability to avert neurologic worsening. In some of these patients, collateral flow is sufficient during the first 3-8 h after onset which averts severe neurologic deficits. However, when collateral failure occurs subsequently, stroke progresses at a time period when advanced tissue injury in the infarct core precludes rescue recanalization therapy. Clinicians are in need of a test to distinguish those collateral-dependent patients who will sustain collateral flow indefinitely and do not require recanalization therapy from those who will experience delayed collateral failure and warrant immediate recanalization interventions while the treatment window is still open.

Diverse clinical and physiologic literature suggests that changes in upright versus supine head and body posture can modify the efficiency of collateral flow in patients with borderline collateral robustness [11-14]. The term 'Heads Down' has been associated with the practice of purposely lowering the head of the bed to increase cerebral blood flow in the ischemic hemisphere [11]. We tested the converse, a 'Heads Up' provocative maneuver with a 90-degree head of bed elevation for $30 \mathrm{~min}$, to stress collaterals and guide early recanalization procedure decision-making in patients with large vessel occlusion or critical stenosis and rapidly improving or mild symptoms while in a supine position.

\section{Methods}

The UCLA Brain Attack team initiated a clinical protocol of performing the Heads Up maneuver in select patients with acute cerebral ischemia to facilitate recanalization decision-making. Criteria for performance of the maneuver were: (1) acute cerebral ischemia within $7.5 \mathrm{~h}$ of onset, (2) initial presentation with a potentially disabling neurologic deficit, (3) spontaneous improvement during acquisition of initial brain imaging to no or a nondisabling neurologic deficit, and (4) evidence from initial time of flight MRA and perfusion MRI of persisting large vessel occlusion. The upper time limit of $7.5 \mathrm{~h}$ was chosen to permit initiation of recanalization therapies within the window cleared by FDA labeling (up to $8 \mathrm{~h}$ for mechanical thrombectomy devices). The judgment of whether a deficit was potentially disabling was made jointly by the senior stroke neurologist and senior neurointerventionalist on clinical grounds; no NIHSS score cutoff or other formal algorithm was employed. 
Patients who met the criteria for Heads Up maneuver performance were brought to the angiography suite and positioned in a trunk and head upright $\left(90^{\circ}\right)$ posture for $30 \mathrm{~min}$. Blood pressure and pulse were measured immediately before and every 5-10 min during upright positioning. If patients neurologically worsened at any point during the 30-min observation period, they were immediately lowered to a supine position and catheter cerebral angiography was promptly initiated. If patients remained neurologically stable throughout the 30-min observation period, they were lowered to a supine or 30-degree head of bed position and transported for admission to the Stroke Unit or Neurocritical Care Unit for medical supportive care. Serial NIHSS were performed to document neurologic course and final outcomes at hospital discharge. Study performance was from April 2005 to April 2006.

Imaging analyses were conducted employing Medical Image Process, Analysis, and Visualization (MIPAV) software (NIH). Perfusion-weighted imaging (PWI) scans were processed to calculate $\mathrm{T}_{\max }$ images using the single-value decomposition method and an automatic AIF selection algorithm. Diffusion and time-to-peak (TTP) lesions (tissue with $\mathrm{T}_{\max }>2 \mathrm{~s}$ ) were identified initially on axial slices employing the MIPAV semi-automated algorithm and subsequently visually inspected and confirmed or refined by an investigator. Individual slice lesions were multiplied by slice thickness $(7 \mathrm{~mm})$ to yield lesion volumes. Mismatch was considered present if the TTP lesion volume was $\geq 20 \%$ larger than diffusion-weighted imaging (DWI) lesion volume. The UCLA Institutional Review Board approved accessing patient charts to collect data for this report.

\section{Results}

Five patients met the protocol entry criteria from April 2005 to April 2006. Their mean age was 78.4 years (range 65-93). All patients presented with substantial deficits (median NIHSS score 11, range 5-22), but improved while in a supine position during MRI scanning to normal or near-normal (NIHSS score 0-2). Among all ischemic stroke patients during the 1-year study period, these patients represented $2.3 \%(5 / 220)$.

MRA showed proximal M1 middle cerebral artery (MCA) occlusions in 2 patients, distal M1 MCA occlusions in 2 patients and intracranial internal carotid artery (ICA) occlusion in 1 patient. Diffusion-perfusion mismatch was present in all 5 patients, and was substantial in each patient. Diffusion lesion volume averaged $1.7 \mathrm{ml}$ (range 0-4.7), perfusion lesion volume averaged $155 \mathrm{ml}$ (range 69.2-222), and percent of perfusion lesion volume occupied by mismatch tissue averaged 98.9\% (range 97.9-100).

On arrival at the Emergency Department, mean \pm SD systolic blood pressure among the 5 patients was $150 \pm 25 \mathrm{~mm} \mathrm{Hg}$ and mean \pm SD diastolic blood pressure was $74 \pm 16 \mathrm{~mm} \mathrm{Hg}$. No drop in blood pressure or alteration in heart rate was observed during the provocative maneuver in all patients.

Two patients sustained their substantial neurologic improvement during Heads Up testing and were subsequently treated with medical care, which included hyperdynamic therapy with norepinephrine for the first $24 \mathrm{~h}$. They had uneventful subsequent hospital courses and excellent outcome with final discharge NIHSS scores of 0 . Follow-up MRI in both medically treated patients on day 3 after completion of the Heads Up test demonstrated smaller perfusion and diffusion lesion volumes. Day 3 MRA showed interval spontaneous partial MCA recanalization in 1 patient and interval spontaneous complete MCA recanalization in the other.

Three patients showed worsening during the Heads Up maneuver, including recurrent hemiparesis and aphasia at the 6th upright minute, recurrent aphasia at the $23 \mathrm{rd}$ upright minute and recurrent hemineglect at the 15th upright minute. Upon worsening, digital subtraction angiography (DSA) was immediately performed demonstrating mid left M1 occlusion, distal left M1 occlusion, and right ICA critical stenosis, respectively. These 3 patients underwent recanalization therapies with successful recanalization and excellent outcome at discharge (table 1). 
Table 1. Clinical characteristics of the patients

\begin{tabular}{|c|c|c|c|c|c|c|c|c|c|c|c|}
\hline $\begin{array}{l}\text { Age/ } \\
\text { sex }\end{array}$ & $\begin{array}{l}\text { Occluded } \\
\text { vessel }\end{array}$ & $\begin{array}{l}\text { Pretreatment } \\
\text { DWI lesion } \\
\text { volume, ml }\end{array}$ & $\begin{array}{l}\text { Pretreatment } \\
\text { TTP lesion } \\
\text { volume, ml }\end{array}$ & $\begin{array}{l}\text { NIHSS } \\
\text { score on } \\
\text { admission }\end{array}$ & $\begin{array}{l}\text { NIHSS } \\
\text { score } \\
\text { before } \\
\text { Heads Up } \\
\text { test }\end{array}$ & $\begin{array}{l}\text { NIHSS } \\
\text { score } \\
\text { after } \\
\text { Heads Up } \\
\text { test }\end{array}$ & $\begin{array}{l}\text { Recanalization } \\
\text { intervention }\end{array}$ & TICI & $\begin{array}{l}\text { NIHSS } \\
\text { score out- } \\
\text { come at } \\
\text { discharge }\end{array}$ & $\begin{array}{l}\text { LKWT to } \\
\text { Heads Up } \\
\text { test, min }\end{array}$ & $\begin{array}{l}\text { Ischemic stroke } \\
\text { subtype(s) }\end{array}$ \\
\hline $93 / \mathrm{F}$ & L MCA M1 & 0.5 & 133.5 & 5 & 0 & 6 & $\begin{array}{l}\text { Endovascular } \\
\text { thrombectomy }\end{array}$ & 3 & 0 & 145 & Cardioembolic \\
\hline $65 / \mathrm{M}$ & R MCA M1 & 2.7 & 159.4 & 11 & 0 & 0 & None & $\mathrm{N} / \mathrm{A}$ & 0 & 115 & Cardioembolic \\
\hline $85 / \mathrm{M}$ & L MCA M1 & 0.8 & 69.2 & 22 & 2 & 4 & $\begin{array}{l}\text { IV tPA and } \\
\text { endovascular } \\
\text { thrombectomy }\end{array}$ & $2 a$ & 1 & 165 & $\begin{array}{l}\text { Large artery } \\
\text { atherosclerosis }\end{array}$ \\
\hline $76 / \mathrm{M}$ & R MCA M1 & 4.7 & 222.4 & 16 & 1 & 0 & None & $\mathrm{N} / \mathrm{A}$ & 0 & 345 & Cardioembolic \\
\hline$\overline{73 / F}$ & $\begin{array}{l}\text { R cervical } \\
\text { ICA }\end{array}$ & 0.0 & 192.2 & 9 & 0 & 6 & $\begin{array}{l}\text { Angioplasty } \\
\text { and stent }\end{array}$ & 3 & 0 & 303 & $\begin{array}{l}\text { Large artery } \\
\text { atherosclerosis and } \\
\text { cardioembolic }\end{array}$ \\
\hline
\end{tabular}
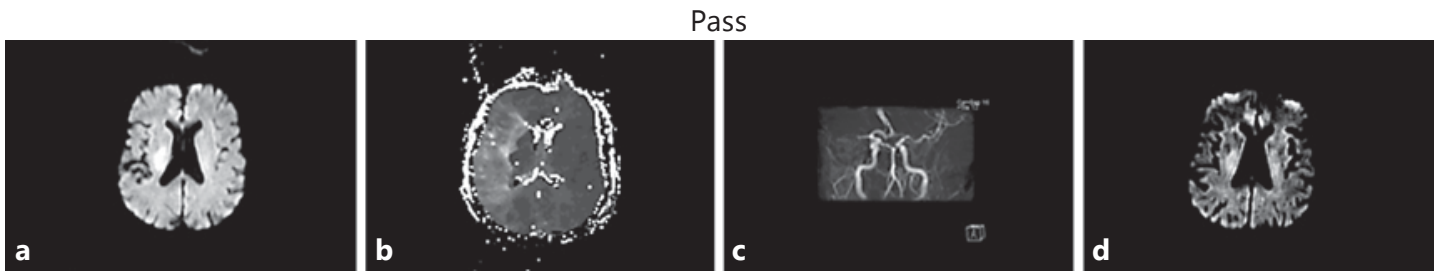

Fig. 1. a Initial DWI showed modest diffusion abnormality in the deep portion of the right MCA territory. b TTP PWI showed large perfusion lesion throughout the right MCA territory, with extensive mismatch. c MRA demonstrated abrupt occlusion of the right M1 segment. After the patient tolerated the Heads Up test, supportive care was pursued. d Follow-up diffusion MRI $3 \mathrm{~h}$ later showed no new diffusion lesions and partial regression of the initial lesion.

\section{Illustrative Cases}

Heads Up Well Tolerated

A 76-year-old man with a history of paroxysmal atrial fibrillation, hypertension, and not on oral anticoagulation presented with left hemiplegia and hemineglect with severe dysarthria (NIHSS score 16). MRA demonstrated abrupt occlusion of the mid-portion of the right horizontal segment of the MCA. MRI demonstrated a 4.7-ml diffusion lesion in the centrum semiovale and a large $222-\mathrm{ml}$ perfusion lesion volume involving the entire superficial right MCA field. On emerging from the scanner, the patient was found to have marked neurologic improvement, with a minimal residual facial weakness (NIHSS score 1). The Heads Up test was performed and the patient did not experience any neurologic worsening throughout the 30-min provocative period. He was then admitted to the neurointensive care unit for supportive care and close monitoring. Repeat imaging $3 \mathrm{~h}$ later showed persistent MCA irregularity in the contour of the right MCA bifurcation and the proximal M2 branches but resolution of the perfusion lesion (fig. 1). The patient's neurologic status was normal at the time of discharge.

\section{Heads Up Provoked Worsening}

A 73-year-old woman with a history of atherosclerotic risk factors and atrial fibrillation, not on oral anticoagulation presented with left-sided hemiparesis, hemisensory neglect and 

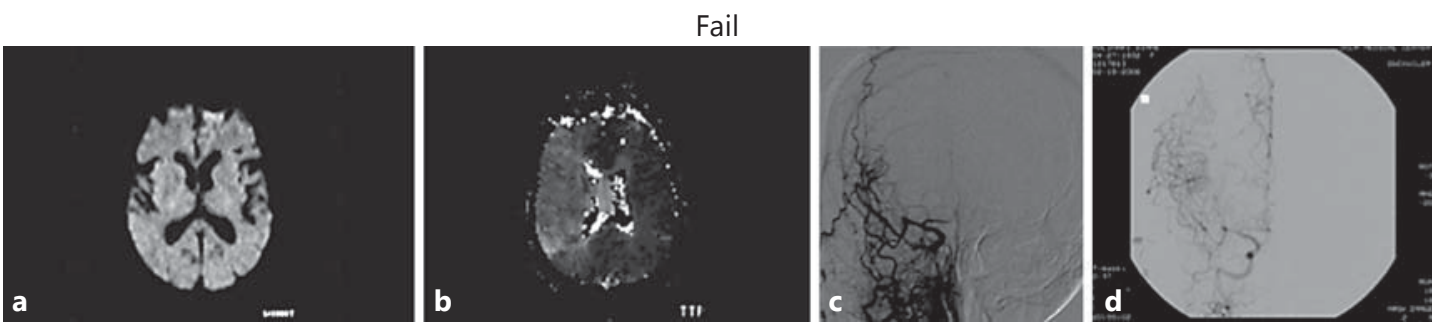

Fig. 2. a DWI demonstrated no acute diffusion lesions. b TTP maps showed reduced flow throughout the right MCA field, with substantial mismatch. c Emergent cerebral angiography demonstrated almost complete occlusion of the right ICA with extensive collateral flow via meningeal branches and retrograde ophthalmic flow to the supraclinoid ICA. d DSA after successful angioplasty and stenting of the proximal right ICA revealed minimal residual stenosis and restoration of the antegrade flow of the right ICA.

dysarthria (NIHSS score 9). Initial MRA showed occlusion or severe stenosis of the right ICA with reconstitution at the ophthalmic artery level. MRI demonstrated no acute diffusion lesions. PWI showed a large 192-ml perfusion lesion volume affecting the entire right MCA territory. The patient improved (NIHSS score 0) after the MRI was completed. While in the angiogram suite, the Heads Up test was performed. The neurological exam worsened to an NIHSS score of 6, with left-sided weakness and hemineglect. The patient was immediately lowered to a supine position with resolution of neurological deficits. DSA revealed nearcomplete occlusion (critical stenosis) of the cervical right ICA with collateral flow to the ICA via the middle meningeal and ophthalmic arteries. Treatment with angioplasty and stenting of the proximal right ICA yielded restoration of antegrade flow within the vessel. The patient's neurologic status was normal after the procedure (fig. 2).

\section{Discussion}

Hemodynamic failure is a major cause of clinical deterioration in acute ischemic stroke $[3,15-17]$. Analyses of tissue state in acute cerebral ischemia typically recognize three regions within a cerebrovascular bed experiencing acutely reduced blood flow: the irreversibly infarcted core; the ischemic 'penumbra', a zone of ischemic tissue in which tissue is viable, but threatened and often not currently functional, and the outer zone of 'benign oligemia' in which blood flow reduction is not threatening tissue viability. In the region of benign oligemia, blood flow rates are reduced below normal but higher than $18 \mathrm{ml} / 100 \mathrm{~g} / \mathrm{min}[18,19]$.

Assessing the adequacy of collateral networks to permanently support peri-infarct regions is a common clinical challenge in acute ischemic stroke patients. Cerebral blood flow rates in the first $48 \mathrm{~h}$ after stroke onset are often dynamic and unstable. A patient may initially have sufficient cardiac output and systemic vascular resistance to mount an adequate rate of flow to peri-infarct regions, but then experience events that challenge collateral networks, including myocardial ischemia, congestive heart failure, dysrhythmias, sepsis, dysautonomia, delayed effects of antihypertensive agents taken prior to stroke onset or other complications that result in reduced cerebral perfusion pressure. Delayed collateral failure can lead to expansion of the infarct core and progression of neurologic deficit [20]. Because baseline resting perfusion images do not directly assess cerebrovascular reserve, they provide only limited information regarding the capacity of peri-infarct fields to withstand further reductions in cerebral perfusion pressure [18]. 
A variety of imaging techniques are used in the nonacute setting to assess cerebrovascular reserve. However, many have drawbacks in the acute setting. Acetazolamide challenge perfusion CT and perfusion MR techniques expose the patient to a prolonged vasodilatory stimulus that may produce vascular steal and increased ischemia in acutely unstable patients. Transcranial Doppler assessment of head position effects upon residual flow through a nearoccluded MCA does not directly assess collateral adequacy when collaterals are the key protection against expansion of the ischemic field should recanalization therapy not be pursued and the nearly-occluded artery becomes totally occluded. Positron emission tomography assessment of oxygen extraction fraction is not generally feasible in acutely ill patients. Compared with these conventional techniques, the Heads Up maneuver has the advantages of simplicity, wide availability, immediate reversibility of the stressor, and positive results (return of neurologic deficit) of incontestable clinical relevance.

The ability of the head position to influence collateral flow has been documented in both clinical series and physiologic investigations. In several case series, assumption of upright head posture was associated with clinical progression of neurologic deficits and, conversely, assumption of head flat posture was associated with clinical resolution of neurologic deficits $[12,13,21]$. More recently, in both transcranial Doppler and diffusion correlation spectroscopy studies, placing patients in a head flat position increased measures of cerebral perfusion by $20 \%$ and was associated with immediate neurologic improvement in $15 \%$ of patients $[11,22]$. A systematic meta-analysis of physiologic studies found that placing acute cerebral ischemia patients in a head flat, rather than elevated, position increases flow velocity in the affected MCA [23]. Retrospective assessment of zero-degree head positioning in the first $24 \mathrm{~h}$ after acute ischemic stroke showed that risk of subsequent aspiration pneumonia is less than $5 \%$ [24]. These findings have led to the recommendation of supine positioning as standard care for patients who can tolerate it [6, 25-29].

Several physiologic factors likely mediate the influence of head position upon cerebral perfusion in cerebral ischemia patients with persisting large vessel occlusion. The cerebral vasculature in the ischemic hemisphere has impaired autoregulation, so that perfusion through collateral channels becomes passive-pressure dependent. Assumption of the upright head position is associated with a reduction in systemic venous return and cardiac output [11].

Our findings are in accordance with the clinical and physiologic literature, and suggest that a 30-min period of heads up positioning may be used as a gentle stressor of collateral systems to identify patients vulnerable to hemodynamic failure. If upright posture alone reduces cerebral perfusion sufficiently to provoke neurological deterioration, then the patient is judged to be at heightened risk of delayed collateral failure, and thrombectomy is immediately pursued.

The initial clinical course in our patients suggested potential positional vulnerability to collateral insufficiency. These 5 acute cerebral ischemia patients all had severe neurologic deficits upon arrival at the Emergency Department but then subsequently improved markedly during MRI scanning with the head flat, despite persistent intracranial arterial occlusion. Once these patients improved, their manifest neurologic deficits were no longer potentially disabling and accordingly they no longer met conventional criteria for pursuing recanalization therapy. However, the persistent intracranial occlusions suggested that at least some of them were at high risk for late hemodynamic failure, stroke progression, and poor final outcome if supportive medical management rather than recanalization therapy was pursued $[5,10,30,31]$. We used head positioning as a tool to gently assess the robustness of collateral flow in these patients. This approach showed promise. The 2 patients who tolerated $30 \mathrm{~min}$ of $90^{\circ}$ head positioning experienced excellent outcomes under medical therapy. Both eventually showed spontaneous recanalization of their large vessel occlusion within $72 \mathrm{~h}$, 
consistent with the known effect of vigorous collaterals in promoting recanalization [32]. The 3 patients who worsened during the heads up maneuver all had successful immediate endovascular recanalization and excellent outcomes.

The 30-min duration of the provocative maneuver was chosen based on two countervailing considerations. A relatively longer interval was desired to provide a challenge that would better index the prolonged period of collateral dependence that would occur under noninterventional management. However, a practical constraint on the duration of the challenge was that, since it was conducted in the angiography suite where immediate intervention can take place if symptoms recur, it ties up personnel away from standard sites of nursing care. Given these considerations, we selected a 30-min interval. Further studies to assess the optimal interval are desirable. As 2 of the 3 provoked worsenings occurred more than $15 \mathrm{~min}$ after maneuver start, shorter durations may be less informative.

This study has several limitations. These observations in a small cohort need to be confirmed and extended in larger samples with more diverse etiologies and longer-term outcomes. Transcranial Doppler assessments of MCA flow velocity during position changes in the studied patients were not obtained. Time of flight MRA was utilized, which is susceptible to misinterpreting slow flow as occlusion. The Heads Up test was conducted in the angiography suite; we would avoid using this test if endovascular rescue therapy is not immediately available. Ultimately, randomized studies are needed to definitively demonstrate that the Heads Up maneuver to select particular patients for intervention improves patient outcome compared with alternative strategies of medical management for all or endovascular management for all.

The Heads Up test may be a useful, simple maneuver to assess the risk of subsequent collateral failure and guide the decision to pursue recanalization therapy in acute cerebral ischemia patients with minimal deficits despite persisting large artery occlusion.

\section{Acknowledgments}

This work was supported by the American Academy of Neurology Clinical Research Fellowship Award.

\section{Disclosure Statement}

S.S. reports grants from Covidien/Medtronic, grants from Stryker Neurovascular, and grants from Genentech outside the submitted work. S.S. is an employee of the University of California. S.S. has served as an unpaid site investigator in multicenter trials run by Medtronic and Stryker for which the UC Regents received payments on the basis of clinical trial contracts for the number of subjects enrolled. The University of California has patent rights in retrieval devices for stroke.

J.L.S. reports contracted payments for services as a scientific consultant from Medtronic, contracted payments for services as a scientific consultant from Stryker, and stock options for services as a scientific consultant from Neuravi outside the submitted work; J.L.S. is an employee of the University of California. J.L.S. has served as an unpaid site investigator in multicenter trials run by Medtronic and Stryker for which the UC Regents received payments on the basis of clinical trial contracts for the number of subjects enrolled. The University of California has patent rights in retrieval devices for stroke.

R.J. reports consulting fees from Medtronic Neurovascular outside the submitted work.

G.R.D. reports consulting fees from Medtronic Medical outside the submitted work.

D.S.L. reports grants from NIH-NINDS, other from Stryker, and other from Medtronic outside the submitted work. 


\begin{tabular}{l|l}
\hline \multicolumn{2}{l|}{ Intervent Neurol 2017;6:8-15 } \\
\hline DOI: 10.1159/000449322 & $\begin{array}{l}\text { C 2016 S. Karger AG, Basel } \\
\text { www.karger.com/ine }\end{array}$ \\
\hline
\end{tabular}

Ali et al.: A Provocative Maneuver to Guide Acute Ischemic Stroke Management

\section{References}

1 Akopov S, Whitman GT: Hemodynamic studies in early ischemic stroke: serial transcranial Doppler and magnetic resonance angiography evaluation. Stroke 2002;33:1274-1279.

2 Kaps M, Teschendorf U, Dorndorf W: Haemodynamic studies in early stroke. J Neurol 1992;239:138-142.

3 Toni D, et al: Progressing neurological deficit secondary to acute ischemic stroke. A study on predictability, pathogenesis, and prognosis. Arch Neurol 1995;52:670-675.

4 Alexandrov AV, et al: Deterioration following spontaneous improvement: sonographic findings in patients with acutely resolving symptoms of cerebral ischemia. Stroke 2000;31:915-919.

5 Rajajee V, et al: Early MRI and outcomes of untreated patients with mild or improving ischemic stroke. Neurology 2006;67:980-984.

6 Jauch EC, et al: Guidelines for the early management of patients with acute ischemic stroke: a guideline for healthcare professionals from the American Heart Association/American Stroke Association. Stroke 2013;44: 870-947.

7 Smith EE, et al: Outcomes in mild or rapidly improving stroke not treated with intravenous recombinant tissuetype plasminogen activator: findings from Get With The Guidelines-Stroke. Stroke 2011;42:3110-3115.

8 Khatri P, et al: Ninety-day outcome rates of a prospective cohort of consecutive patients with mild ischemic stroke. Stroke 2012;43:560-562.

9 Barber PA, et al: Why are stroke patients excluded from TPA therapy? An analysis of patient eligibility. Neurology 2001;56:1015-1020.

10 Nedeltchev K, et al: Outcome of stroke with mild or rapidly improving symptoms. Stroke 2007;38:2531-2535.

11 Wojner-Alexander AW, et al: Heads down: flat positioning improves blood flow velocity in acute ischemic stroke. Neurology 2005;64:1354-1357.

12 Caplan LR, Sergay S: Positional cerebral ischaemia. J Neurol Neurosurg Psychiatry 1976;39:385-391.

13 Dobkin BH: Orthostatic hypotension as a risk factor for symptomatic occlusive cerebrovascular disease. Neurology 1989;39:30-34.

14 Bang OY, et al: Collateral circulation in ischemic stroke: assessment tools and therapeutic strategies. Stroke 2015;46:3302-3309.

15 Toni D, et al: Acute ischemic strokes improving during the first 48 hours of onset: predictability, outcome, and possible mechanisms. A comparison with early deteriorating strokes. Stroke 1997;28:10-14.

16 Toni D, et al: Early spontaneous improvement and deterioration of ischemic stroke patients. A serial study with transcranial Doppler ultrasonography. Stroke 1998;29:1144-1148.

17 Alawneh JA, Moustafa RR, Baron JC: Hemodynamic factors and perfusion abnormalities in early neurological deterioration. Stroke 2009; 40:e443-e450.

18 Dani KA, et al: Computed tomography and magnetic resonance perfusion imaging in ischemic stroke: definitions and thresholds. Ann Neurol 2011;70:384-401.

19 Bandera E, et al: Cerebral blood flow threshold of ischemic penumbra and infarct core in acute ischemic stroke: a systematic review. Stroke 2006;37:1334-1339.

20 Tisserand $\mathrm{M}$, et al: Mechanisms of unexplained neurological deterioration after intravenous thrombolysis. Stroke 2014;45:3527-3534.

21 Sharma VK, et al: Thrombotic occlusion of the common carotid artery (CCA) in acute ischemic stroke treated with intravenous tissue plasminogen activator (TPA). Eur J Neurol 2007;14:237-240.

22 Favilla CG, et al: Optical bedside monitoring of cerebral blood flow in acute ischemic stroke patients during head-of-bed manipulation. Stroke 2014;45:1269-1274.

23 Olavarria VV, et al: Head position and cerebral blood flow velocity in acute ischemic stroke: a systematic review and meta-analysis. Cerebrovasc Dis 2014;37:401-408.

24 Palazzo P, et al: Risk of pneumonia associated with zero-degree head positioning in acute ischemic stroke patients treated with intravenous tissue plasminogen activator. Brain Behav 2016;6:e00425.

25 Shafi N, Kasner SE: Treatment of acute ischemic stroke: beyond thrombolysis and supportive care. Neurotherapeutics 2011;8:425-433.

26 Diserens K, Michel P, Bogousslavsky J: Early mobilisation after stroke: review of the literature. Cerebrovasc Dis 2006;22:183-190.

27 Burns JD, et al: Intensive care management of acute ischemic stroke. Emerg Med Clin North Am 2012;30: 713-744.

28 Middleton S, Grimley R, Alexandrov AW: Triage, treatment, and transfer: evidence-based clinical practice recommendations and models of nursing care for the first 72 hours of admission to hospital for acute stroke. Stroke 2015; 46:e18-e25.

29 Glober NK, et al: Acute stroke: current evidence-based recommendations for prehospital care. West J Emerg Med 2016;17:104-128.

30 Kim JT, et al: Proximal arterial occlusion in acute ischemic stroke with low NIHSS scores should not be considered as mild stroke. PLoS One 2013;8:e70996.

31 Brown TA, et al: Magnetic resonance imaging in acute ischemic stroke patients with mild symptoms: an opportunity to standardize intravenous thrombolysis. J Stroke Cerebrovasc Dis 2015;24:1832-1840.

32 Calleja AI, et al: Collateral circulation on perfusion-computed tomography-source images predicts the response to stroke intravenous thrombolysis. Eur J Neurol 2013;20:795-802. 